\title{
The Amino Acid Permeases AAP3 and AAP6 Are Involved in Root-Knot Nematode Parasitism of Arabidopsis
}

\author{
Heather H. Marella, Erik Nielsen, Daniel P. Schachtman, and Christopher G. Taylor \\ Donald Danforth Plant Science Center, 975 N. Warson Rd., St. Louis, MO 63132, U.S.A.
}

Submitted 20 May 2012. Accepted 16 September 2012.

\begin{abstract}
The root-knot nematode, Meloidogyne incognita, is an obligate parasite which depends entirely on the host plant for its nutrition. Root-knot nematodes induce the formation of a highly specialized feeding site consisting of several giant cells surrounded by a network of vascular tissues. Nutrients, including amino acids and sugars, are transferred apoplastically from the vascular tissues to the feeding site. Using Arabidopsis thaliana lacking the vascular-expressed amino acid permeases (AAP) AAP3 or AAP6, we demonstrate that disruption of amino acid transport can affect nematode parasitism. Nematode infestation levels are significantly reduced on the aap3 and aap6 mutants. AAP3 and AAP6 act distinctly in the transport of amino acids to the feeding site, as demonstrated by differences in their carrying capacity profiles. Furthermore, analyses of promoter: $\beta$-glucuronidase lines show different expression patterns for $A A P 3$ and $A A P 6$ in infected roots. In the aap3-3 mutant, part of the decrease in infestation is connected to a defect in early infection, where juveniles enter but then leave the root. Both aap3-3 and aap6-1 produce fewer females and produce more adult male nematodes. Additionally, detrimental effects are observed in the nematodes harvested from aap3-3 and aap6-1 mutants, including decreased egg hatching and infectivity and lower levels of lipid reserves. The transport of amino acids by AAP3 and AAP6 is important for nematode infection and success of the progeny.
\end{abstract}

The root-knot nematode (RKN) Meloidogyne incognita, an obligate parasitic nematode, is among the most devastating of plant pathogens. Capable of reproducing on over 2,000 species of plants, RKN cause the formation of large knot-like struc-

Current address for H. H. Marella: Bridgewater State University, Department of Biological Sciences, Conant Science and Math Center, 24 Park Ave., Bridgewater, MA 02325, U.S.A.

Current address for E. Nielsen: University of Michigan, Molecular, Cellular, and Developmental Biology, Natural Science Building, 830 North University Ave., Ann Arbor 48109, U.S.A.

Current address for D. P. Schachtman: Monsanto Company, 700 Chesterfield Parkway West, Chesterfield, MO 63017, U.S.A.

Current address for C. G. Taylor: Ohio Agricultural Research and Development Center, Ohio State University, Department of Plant Pathology, 1680 Madison Ave., Wooster 44691, U.S.A.

Corresponding author: C. G. Taylor; E-mail: taylor.1886@osu.edu

This article is in the public domain and not copyrightable. It may be freely reprinted with customary crediting of the source. The American Phytopathological Society, 2013. tures in the infected roots, disrupting the development and nutrient flow of the root system (Bird 2004; Caillaud et al. 2008; Williamson and Gleason 2003). Infection begins with a second-stage juvenile (J2) RKN entering the root behind the tip or at sites of lateral root initiation, and then migrating up the central cylinder until it establishes a feeding site. Upon the formation and development of a feeding site, the $\mathrm{J} 2$ becomes sedentary and undergoes several molts before turning into an adult. Development into an adult female is the default pathway for RKN, which can produce a thousand eggs through mitotic parthenogenesis. However, under certain conditions, such as host stress or nutrient deprivation, adult males will develop, abandon their feeding sites, and leave the root as a mechanism for maintaining the optimal parasitic load for the survival of the population (Davide and Triantaphyllou 1967, 1968; Snyder et al. 2006).

RKN establish a highly specialized feeding site consisting of several giant cells that are differentiated from cells in the stele region of the root. These cells enlarge into giant cells (many times the size of typical root xylem parenchyma cells), contain multiple nuclei, and have thickened and highly invaginated cell wall regions. The RKN rely entirely on these giant cells to provide all of their dietary requirements, including water, carbohydrates, and amino acids. In the RKN parasitism process, the giant cells become major nutrient sinks in the plant (Caillaud et al. 2008).

Given that nematodes require amino acids in their diet, particularly the essential amino acids which they cannot synthesize, it is expected that amino acid transporters would be enriched in the giant cells and also the tissues supplying these feeding cells. In Arabidopsis, there are over 60 amino acid transporter genes, which have been divided into several families based on protein phylogeny (Chang et al. 2004; Rentsch et al. 2007; Wipf et al. 2002). Most of the amino acid transporters fall into two families: the amino acid/auxin permease (AAAP) family and the amino acid-polyamine-choline (APC) family. The majority of the research on these transporters has focused on the members of the AAAP family and has defined roles for some of these transporters in the import of amino acids into seed and phloem and uptake of amino acids in by the root (Tegeder 2012). The AAAP family consists of six subfamilies, of which the eight members of the amino acid permease subfamily (AAP1-8) are best characterized in terms of expression, transport profile, and function (Tegeder and Rentsch 2010), which made the AAP family an attractive group of transporters to examine for roles in nematode parasitism.

Expression profiling of whole roots of Arabidopsis thaliana infected with RKN (Hammes et al. 2005; Jammes et al. 2005) and laser-microdissected giant cells and galls (Barcala et al. 2010) identified many transporter genes that were differentially regulated upon nematode infection. Among these differ- 
entially regulated transporters were several amino acid transporters, including the amino acid permeases $A A P 1, A A P 3$, $A A P 6$, and $A A P 7$, which were upregulated during infection with RKN (Barcala et al. 2010; Hammes et al. 2005). In wholeinfected root experiments, expression of $A A P l$ was induced at all three time points tested (1,2, and 4 weeks postinoculation) while the other three genes ( $A A P 3, A A P 6$, and $A A P 7)$ were upregulated at the 2-week time point (Hammes et al. 2005). AAP6 was highly expressed in the giant-cell-containing knots, as tested by real-time reverse-transcription polymerase chain reaction (RT-PCR) and pAAP6: $\beta$-glucuronidase $(G U S)$ plants, confirming the microarray results (Hammes et al. 2005). AAP6 expression was also induced by infection of the beet cyst nematode, Heterodera schachtii, in Arabidopsis and by soybean cyst nematode, $H$. glycines, on soybean (Puthoff et al. 2003, 2007). Additionally, the expression of both AAP3 and $A A P 6$ is upregulated in the syncytia formed during $H$. schachtii infection of Arabidopsis (Szakasits et al. 2009).

The function of AAP3 and AAP6 has been characterized in Xenopus oocytes and yeast (Fischer et al. 1995, 2002; Okumoto et al. 2002). Both AAP3 and AAP6 transport a broad spectrum of amino acids, including several essential amino acids, but differ in their specificities for many amino acids. AAP3 can transport basic amino acids while AAP6 transports aspartate with high affinity. Both $A A P 3$ and $A A P 6$ are expressed in roots as well as in aerial tissue and are localized to the vasculature, as determined by promoter:GUS fusions. In roots, AAP3 is localized to phloem companion cells whereas AAP6 is localized to xylem parenchyma cells (Okumoto et al. 2002, 2004). Based on these localization patterns, it is believed that these transporters are involved in the long-distance transport of amino acids. Additionally, it was demonstrated that the sieve element sap of an aap6 mutant had reduced overall amino acid content, with significantly less aspartic acid, leucine, lysine, and phenylalanine (Hunt et al. 2010).

Because $A A P 3$ and AAP6 show differential expression during RKN infestation, we investigated the role of these amino acid permeases during the infection of Arabidopsis by RKN. Here, we demonstrate that RKN infestation is reduced on T-DNA insertion lines for which $A A P 3$ (aap3-3) or AAP6 (aap6-1) are inactivated. The decrease in infestation is linked to an impairment of early infection in the aap3-3 mutant and increased adult male production on both aap3-3 and aap6-1. Furthermore, we show that the effect of feeding on aap3-3 and aap6-1 is carried to the next generation of $\mathrm{RKN}$, which display reduced egg hatching and infection along with a decrease in lipid reserves. These results establish a link between the host amino acid transport system and the nutritional requirements of RKN in the compatible parasitic interaction.

\section{RESULTS}

Nematode infection is reduced on aap3 and aap6 mutants.

In order to determine whether the amino acid permeases whose expression is altered under nematode infestation are important during nematode parasitism, we tested knockout mutants for $A A P 3$ and AAP6. The aap3-3 and aap6-1 mutants were isolated from the Salk T-DNA collection (Fig. 1A) and the absence of transcript was confirmed by RT-PCR (Fig. 1B). The aap3-2 (Okumoto et al. 2004) mutant has been previously described.

Because differences in the root morphology could affect RKN infestation levels, we examined the root architecture of the aap3, aap6, and aap3-3/aap6-1 mutants. The aap3 mutants and the aap3-3/aap6-1 double mutant produce an enlarged root system with a longer primary root with more lateral roots than either wild-type or aap6-1 mutant plants. Roots of 21day-old aap3-3 mutant seedlings have 37\% more lateral roots (Fig. 2A) and a 14\% longer primary root (Fig. 2B) than the Columbia wild-type control. Similar changes in root architecture are observed for the aap3-2 mutant relative to the Ws wild-type (Fig. 2A and B). No changes in root architecture were observed for aap6-1 plants (Fig. 2A and B). The aap3-3/ aap6-1 double mutant exhibits a larger root system similar to that of the aap3-3 mutant alone (Fig. 2A and B).

Homozygous aap3-2, aap3-3, and aap6-1 mutants along with their respective wild type were inoculated with RKN eggs and the resulting number of adult females producing egg masses was counted as a measure of infestation level. The aap3-2 and aap3-3 mutants had a 26 and $29 \%$ reduction, respectively, in
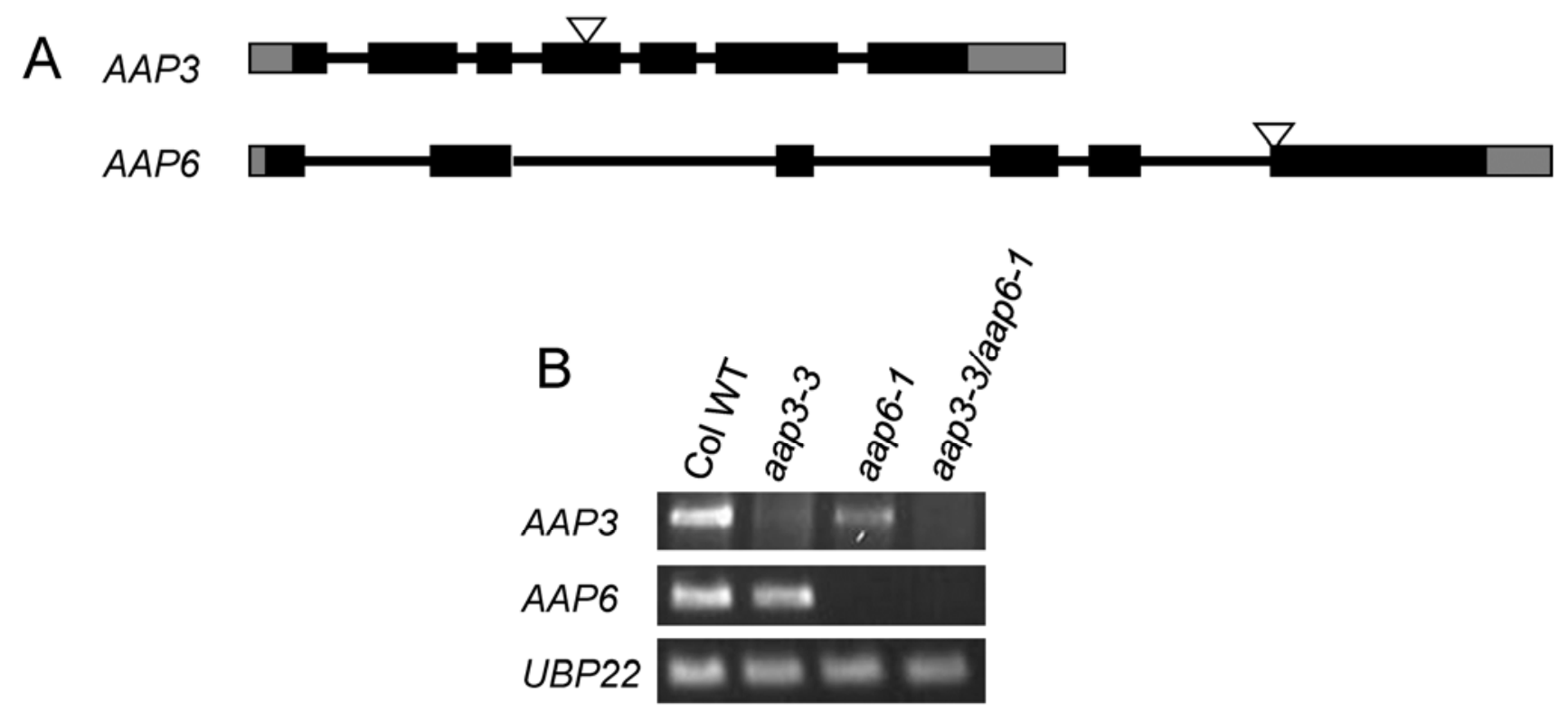

Fig. 1. Molecular characterization of amino acid permease $(A A P) 3$ and $A A P 6$ T-DNA insertion lines. A, Schematic diagrams of the genomic loci of $A A P 3$ and $A A P 6$. Exons are represented as black boxes, the untranslated regions as gray boxes, and the introns as black bars. The site of T-DNA integration is marked with a triangle. B, Reverse-transcription polymerase chain reaction analysis of $A A P 3$ and $A A P 6$ in the single- and double-mutant lines shows a lack of transcript in the mutants due to the T-DNA insertion. AtUBP22 (At5g10790) is used as a positive control. 
the number of adult females with egg masses, while the aap6-1 mutant had a $38 \%$ reduction (Fig. 3). We generated a homozygous aap3-3/aap6-1 double mutant line, confirmed by RTPCR, to test whether there would be a greater reduction in nematode infestation (Fig. 1B). The aap3-3/aap6-1 double mutant displayed a reduction in egg masses similar to that of the individual aap3-3 and aap6-1 mutants (Fig. 3). This result shows a lack of an additive or synergistic effect of the mutations, possibly indicating that these amino acid permeases are acting in series to move amino acids toward the feeding sites. All subsequent experiments were carried out with the single aap3-3 and aap6-1 mutants because the double mutant did not display enhanced nematode resistance and because the aap3-2 mutant is in the Ws wild-type background for which we did not have a corresponding aap6 mutant.

Because we observed a reduction in egg mass number on the mutants for $A A P 3$ and $A A P 6$, we decided to determine whether the nematode carrying capacity (i.e., the number of females that it could support) of these mutants is also decreased. Increasing amounts of RKN J2 were added to aap3-3, aap6-1, and Columbia wild-type seedlings and the resulting number of adult females with egg masses was counted. The aap6-1 mutant and wild-type plants supported equal numbers of egg masses at the lowest inoculation level but the carrying capacity of aap6-1 reached a peak carrying capacity of between 600 and $900 \mathrm{~J} 2$, resulting in a significantly lower number of egg masses supported as compared with the wild type (Fig. 4). The carrying capacity of the aap3-3 mutant was consistently lower than that of the wild type even at the lowest level of J2 inoculation (Fig. 4). These data suggest that the aap3-3 and aap6-1 mutants may lead to a reduction in nematode infection in distinct ways.

\section{The expression of AAP3 and AAP6 differ in $\mathrm{RKN}$ infected roots.}

To determine whether the expression pattern of $A A P 3$ and $A A P 6$ might contribute to the differences in carrying capacity between the mutants, we analyzed the expression pattern of these amino acid permeases at different time points during RKN infection using promoter:GUS transgenic lines for AAP3 (Okumoto et al. 2004) and AAP6 (Hammes et al. 2005). One week after inoculation, both the pAAP3:GUS $S_{L}$ and $\mathrm{pAAP6:GUS}$ lines showed expression in infected root tips penetrated by RKN as well as the primary root vasculature, whereas the neighboring uninfected (not penetrated) root tips of the same seedling remained unstained (Fig. 5A and E). These results indicate that expression of both $A A P 3$ and $A A P 6$ is induced early on in the infected root tissue. Four weeks after inoculation, distinct differences were seen between the expression patterns of $A A P 3$ and $A A P 6$. High levels of $A A P 3$ expression are observed in the root vasculature, especially near the galls (Fig. 5C), but low levels of expression are observed in the gall itself (Fig. 5D). Microscopic observation of sectioned galls confirms the low level of $A A P 3$ expression, including the giant cells (Fig. 5B). In contrast, the expression of $A A P 6$ at 4 weeks of infection is very strong in the galls (Fig. $5 \mathrm{~F}$ ). We have previously shown that $A A P 6$ is expressed in the giant cells using

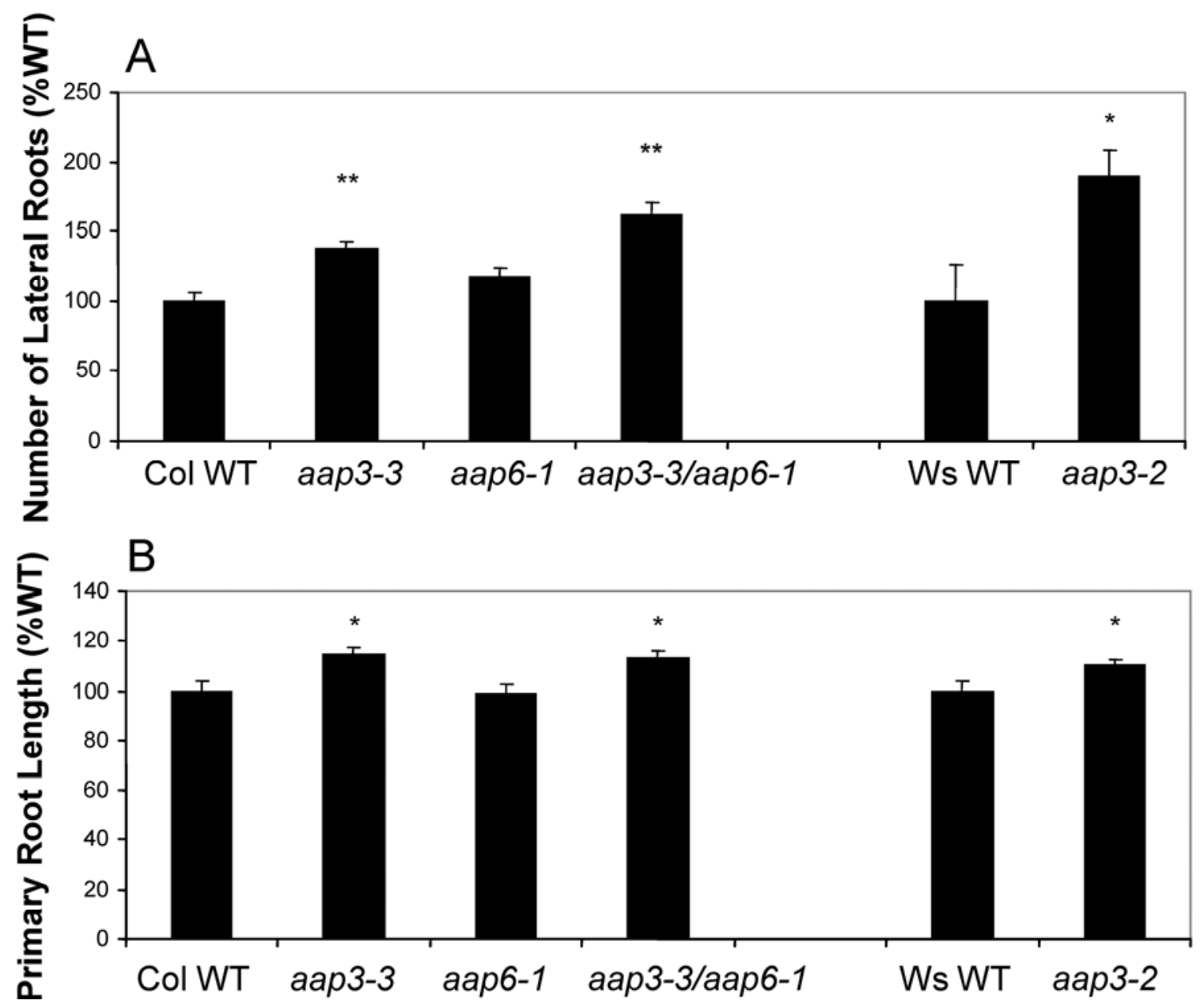

Fig. 2. Assessment of root architecture in amino acid permease mutants. Eighteen seedlings were monitored for root length and number of lateral roots for the entire primary root over the course of 21 days. A, Number of lateral roots and $\mathbf{B}$, primary root length as measured on day 21 . $P$ values were calculated using the Student's $t$ test. ( $*$ and $* *=P<0.05$ and $<0.001$, respectively). Col WT $=$ Columbia wild type and aap $=$ amino acid permease. 
sections of this same transgenic line (Hammes et al. 2005). These expression pattern differences provide additional support for $A A P 3$ and $A A P 6$ acting in distinct manners to move amino acids into the nematode feeding site.

\section{Juvenile retention is lower for the aap3-3 mutant.}

Because the number of infection sites may be important for nematode infestation of roots, we normalized the number of available root tips for infection. Seven-day-old aap3-3, aap6-1, and Columbia wild-type seedlings, all with only a single primary root, were inoculated with $\mathrm{J} 2$. Nematode infection was monitored every 2 days by acid fuchsin staining of the J2 inside each root. On day 2, the aap3-3 mutant had significantly more $\mathrm{J} 2$ inside of the root than the wild-type control (Fig. 6A). However, by day 6, the aap3-3 mutant had significantly fewer $\mathrm{J} 2$ inside the roots than the wild-type and aap6-1 mutant plants (Fig. 6A). In fact, there was no increase in the number of $\mathrm{J} 2$ in the roots of aap3-3 mutants between day 4 and day 6 .

A similar assay was conducted to determine why there were fewer $\mathrm{J} 2$ in the roots of the aap3-3 mutant on the sixth day, because the $\mathrm{J} 2$ could either be leaving the root or be impaired in entering the root. Seedlings ( 7 days old) were infected with J2 and kept on the infection plate for 4 days because, on day 4 , we did not observe any difference in the number of $\mathrm{J} 2$ inside the roots of aap3-3, aap6-1, or the wild-type seedlings (Fig. 6A). Each seedling was then placed in an individual well of Pluronic gel, which solidified around the root. After another 2 days, the seedling was removed and stained with acid fuchsin to visualize the $\mathrm{J} 2$ inside the root. The number of escaping $\mathrm{J} 2$ left behind in each well of Pluronic gel was counted to determine the percentage of $\mathrm{J} 2$ retained in each root. The aap3-3 mutant retained significantly fewer infective $\mathrm{J} 2$ than the Columbia wild-type or aap6-1 mutant (Fig. 6B). The 27\% reduction of $\mathrm{J} 2$ retention in the aap3-3 mutant indicates that the early stages of the nematode infection process may be altered in the aap3-3 mutant plant.

\section{Development of adult male nematodes is increased in the aap3-3 and aap6-1 mutants.}

We examined whether the number of adult male nematodes that developed during the course of the nematode bioassay differed on aap3-3 and aap6-1 plants. Seedlings of aap3-3, aap6-1, and Columbia wild-type were inoculated with RKN eggs. After 6 weeks, the media and root material was collected from each plate and melted down in the presence of acid fuchsin to stain for nematodes. All nematodes were collected by filtration and the number of adult males counted. Both aap3-3 and aap6-1 mutants produce more adult male nematodes (five to six times more, respectively) relative to the wildtype control (Fig. 7). This switch from female to male development is indicative of suboptimal host conditions (Davide and Triantaphyllou 1967, 1968; Snyder et al. 2006).

\section{Nematodes propagated on the aap3 and aap6 mutant plants} display reduced vigor and reduced lipid reserves.

In order to determine whether nematode propagation on the aap3-3 and aap6-1 mutants would affect the next generation of nematodes, we examined the nematode progeny produced on these mutants. Individual adult females along with their egg masses were isolated from aap3-3, aap6-1, and Columbia wildtype plants and placed in Pluronic gel. The hatching and emergence of $\mathbf{J} 2$ from each egg mass was monitored. Egg number and egg and juvenile morphology appeared normal on the aap3-3 and aap6-1 plants (data not shown). However, threefold fewer $\mathbf{J} 2$ hatched and emerged from the egg mass for females isolated from both the aap3-3 and aap6-1 mutants (Fig. 8).

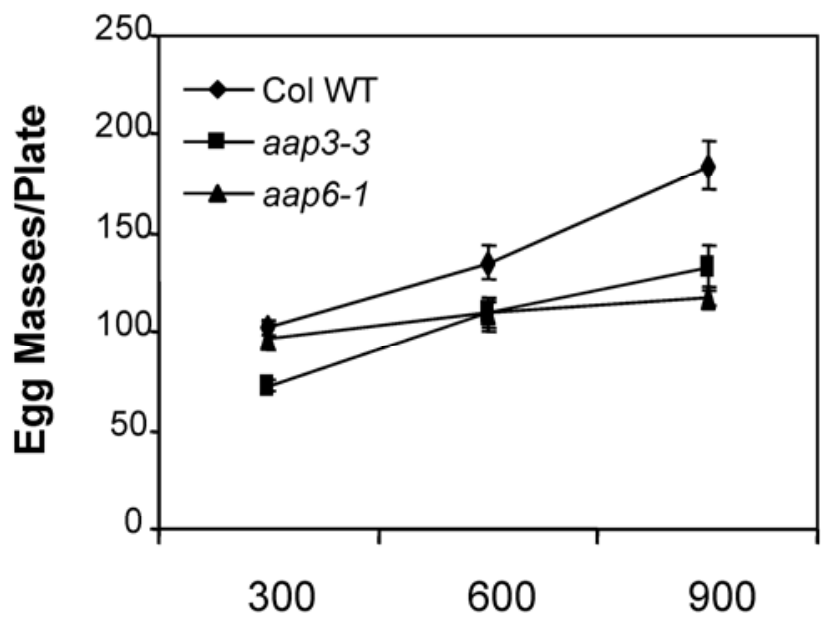

\section{Number of J2 Inoculated}

Fig. 4. Evaluation of root-knot nematode carrying capacity in Columbia wild type (Col WT), amino acid permease (aap)3-3, and aap6-1. Plates of Col WT, aap3-3, and aap6-1 with five plants per plate and eight plates per trial were inoculated with increasing amounts of second-stage juveniles (J2). After 7 weeks, the number of adult females with egg masses was counted for each plate. Error bars indicate standard error.

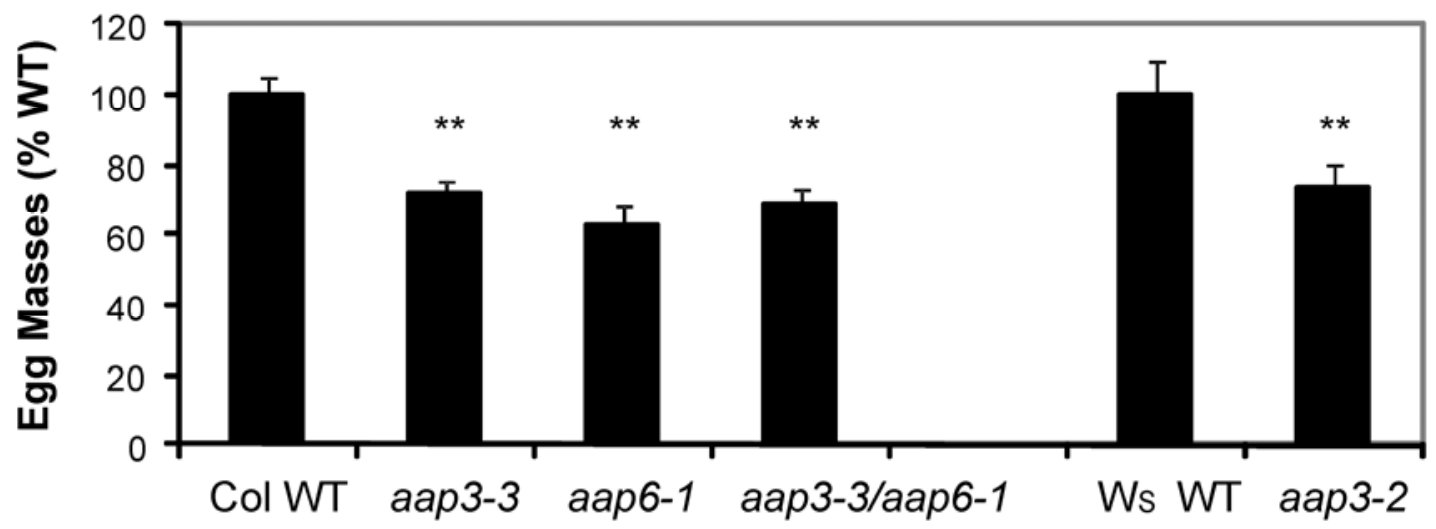

Fig. 3. Comparison of root-knot nematode infestation in amino acid permease (aap) mutants and the wild type (WT). Five Arabidopsis plants on a single plate, with 10 plates per trial, were inoculated with 1,000 root-knot nematode (RKN) eggs. After 8 weeks, the number of females producing an egg mass was counted for each plate. Data are normalized as a percentage of the respective WT value. The reduction in infestation level for the aap3, aap6, and aap3-3/aap6-1 mutants is significant relative to the respective WT ( $* *=P<0.001)$ as calculated using the Student's $t$ test. The number of independent experiments $(n)$ conducted for each line: Columbia (Col) WT $(n=7)$, aap3-3 $(n=4)$, aap6-1 $(n=6)$, aap3-3/aap6-1 $(n=3)$, Ws WT $(n=3)$, and aap3-2 $(n=3)$. 
Because we observed reduced hatching from egg masses, we investigated whether this reduced vigor also affected the infectivity of $\mathrm{J} 2$ propagated on the aap3-3 and aap6-1 mutants. Using synchronized hatches of $\mathbf{J} 2$ from eggs harvested from aap3-3, aap6-1, and the Columbia wild type, tobacco plants were infected with $200 \mathrm{~J} 2$ per plant. We chose to use sandgrown tobacco as the host because it allowed us to test infectivity in a more realistic setting, rather than using a plate-based assay, and the sand rinsed easily away to visualize RKN egg masses. The number of resulting adult females producing egg masses was assessed by Phloxine B staining the tobacco roots at 7 weeks postinoculation. Juveniles propagated from aap3-3 (Fig. 9A) and aap6-1 (Fig. 9B) displayed a 40 and 55\% reduction in infection, respectively. The reduced vigor of the progeny from aap3-3 and aap6-1 mutants suggests that disrupted transport of amino acids is detrimental to nematode parasitism.
On seeing a reduction in the vigor of juveniles propagated on the aap3-3 and aap6-1 plants, we examined the lipid energy reserves of the juveniles. We stained $\mathbf{J} 2$ collected from Columbia wild-type, aap3-3, and aap6-1 plants with the neutral lipid dye Nile Red. Using confocal microscopy, we observed less Nile Red staining of the intestinal lipid stores of the juveniles from aap3-3 and aap6-1 plants. Juveniles from the Columbia wild-type plants had brightly stained, round lipid droplets filling their intestine (Fig. 10A and D). However, the intestines of the juveniles from aap6-1 (Fig. 10B and E) and aap3-3 (Fig. 10C and F) plants had less Nile Red staining, with large gaps where lipid droplets should be visible. Of the $\mathrm{J} 2$ examined for each line, only $38 \%$ of aap3-3- and $28 \%$ of aap6-1-propagated nematodes had intestines filled with NileRed-stained lipid droplets as compared with $80 \%$ of the $\mathrm{J} 2$ propagated on the Columbia wild type. These results indicate
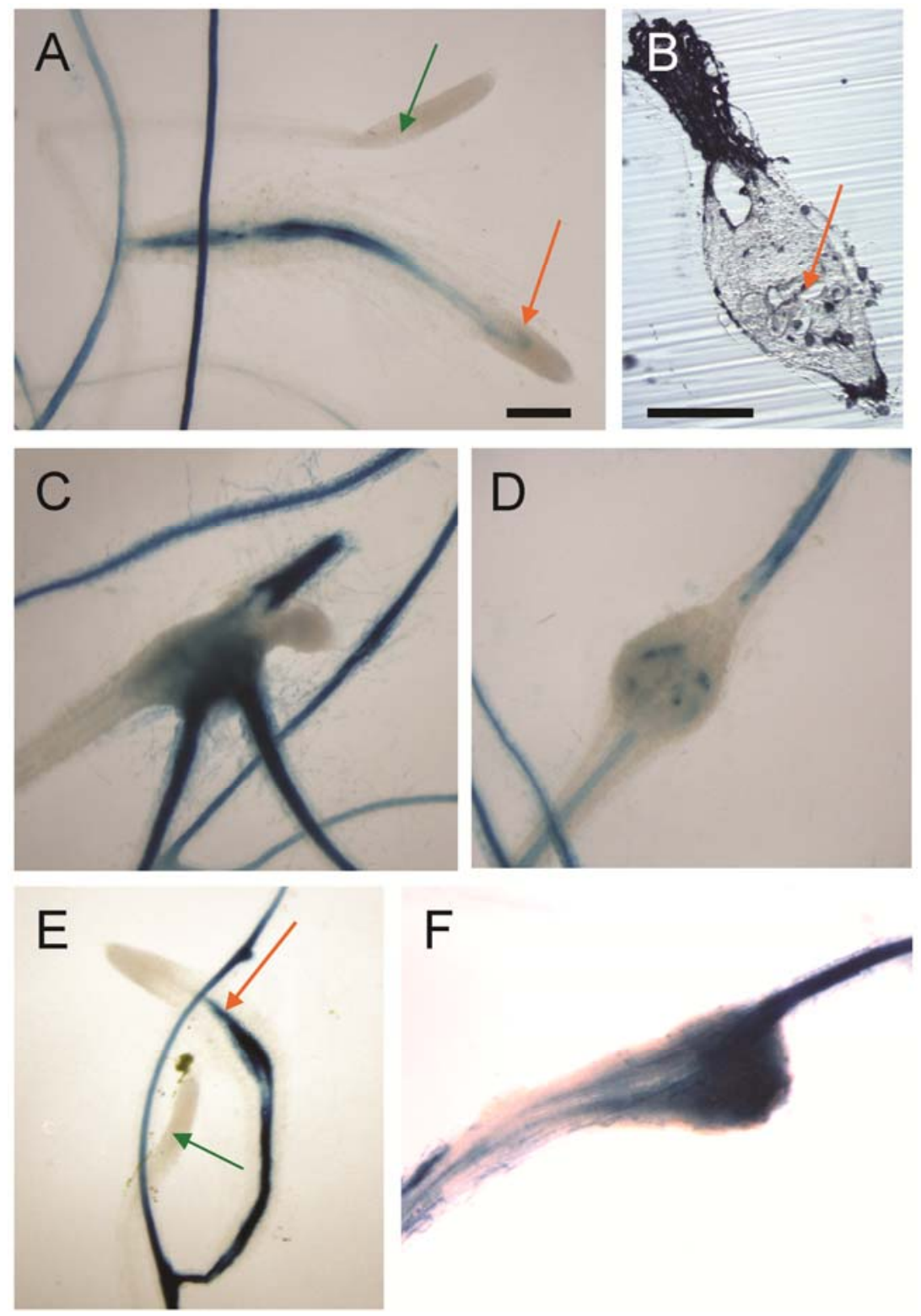

Fig. 5. Histochemical localization of amino acid permease $(A A P) 3$ and $A A P 6$ expression in root-knot nematode (RKN)-infected roots. Three-week-old pAAP3:GUS $S_{L}$ and pAAP6:GUS Arabidopsis plants were inoculated with RKN eggs. $\beta$-Glucuronidase (GUS) staining was performed at $\mathbf{A}$ and $\mathbf{E}, 1$ week and $\mathbf{B}$ to $\mathbf{D}$ and $\mathbf{F}, 4$ weeks postinoculation. At 1 week postinoculation, GUS expression for both $\mathbf{A}, \mathrm{p} A A P 3: G U S_{L}$ and $\mathbf{E}, \mathrm{pAAP} 6: G U S$ is seen in the RKN-infected root tips (orange arrows) as well as the vasculature of the primary root. Uninfected lateral roots are indicated with green arrows. C and D, At 4 weeks postinoculation, GUS expression for the pAAP3:GUS $S_{L}$ is very high in the root vasculature but low in the knots. Wax-embedded sections of pAAP3:GUS ${ }_{L}$ knots show very little GUS staining, with a lack of staining seen in the giant cells (orange arrow); $\mathbf{B}$, however, GUS staining is seen in the root tissue adjacent to the knot. F, At 4 weeks postinoculation, GUS expression for the pAAP6:GUS is very high in the root vasculature and also in the knots. Scale bars represent $200 \mu \mathrm{m}$. 
that the lipid reserves are altered in the nematodes propagated on the aap3-3 and aap6-1 mutants, possibly leading to the observed lack of vigor.

\section{DISCUSSION}

RKN alter the expression of several amino acid transporters in infected Arabidopsis roots (Barcala et al. 2010; Hammes et al. 2005; Jammes et al. 2005). Among these, AAP3 and AAP6 influence the success of nematode parasitism, as demonstrated through the use of mutant plants. Amino acid transporters are central to infection for several other obligate pathogens such as fungal, protozoan, and insect parasites. The rust fungus Uromyces fabae induces expression of a general amino acid permease, UtAAT3, in haustoria to acquire amino acids from the leaf apoplast (Struck et al. 2004). The expression of the cationic amino acid transporter $C A T 2 B$ from mouse is upregulated during Leishmania amazonensis infection, thereby providing the arginine necessary for proliferation of the parasite (Wanasen et al. 2007). The aromatic and neutral amino acid transporter AtANT1 affects the aphid-Arabidopsis relationship by influencing the ingestion rate of the aphid (Hunt et al. 2006). AAP6 also has slight effects on aphid parasitism as measured by increased salivation and reduced reproduction (Hunt et al. 2010). The more severe effects we observe for RKN are most likely due to the type of tissue infected (roots

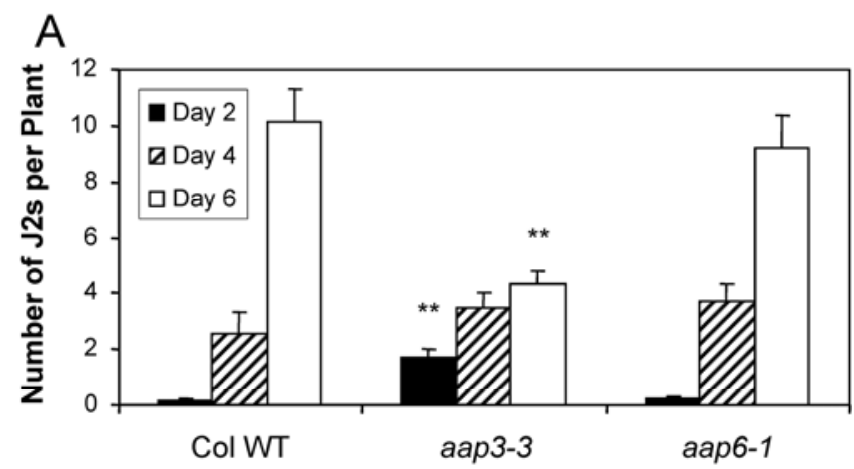

$\mathrm{B}$

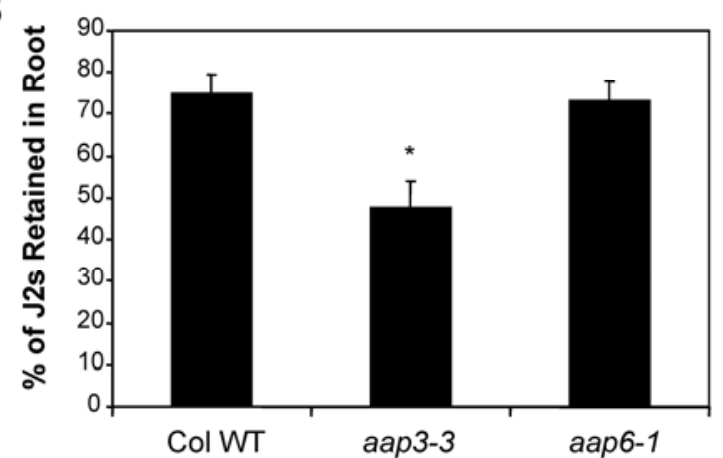

Fig. 6. Comparison of the early stages of root-knot nematode (RKN) infection in the Columbia wild type (Col WT) and amino acid permease (aap)3-3 and aap6-1 mutants. Twenty seedlings, each with only a primary root, were infected with 1,000 RKN second-stage juveniles (J2) for both A and B. A, Acid fuchsin staining of RKN inside of the roots over a time course at 2, 4, and 6 days after infection. Significant alterations in the number of juveniles in the aap3-3 mutant were seen on day 2 and day 6 compared with the Col WT. B, After 4 days of infection by the RKN J2, the seedlings were moved into individual wells of Pluronic gel to capture any $\mathrm{J} 2$ leaving the root for the next $48 \mathrm{~h}$. The number of $\mathrm{J} 2$ in the Pluronic gel and those stained by acid fuchsin inside of the root were counted. Significantly fewer juvenile nematodes were retained in the aap3-3 mutant than the Col WT. $P$ values were calculated using the Student's $t$ test. (* and $* *=P<0.05$ and $<0.001$, respectively). for RKN versus aerial tissue for aphids) and the fact that RKN establish giant cells to feed from rather than directly tapping into the phloem like aphids.

The role of amino acid transporters in nematode infection.

Obligate plant-parasitic nematodes such as RKN require the uptake of amino acids, including the essential amino acids, from the host plant. During the nematode infestation process, numerous amino acid transporters are induced in the feeding sites as well as in the rest of the infected roots (Barcala et al. 2010; Hammes et al. 2005; Jammes et al. 2005). Giant cells, which lack plasmodesmata and are symplastically isolated, collect or produce the necessary nutrients that nematodes withdraw for their growth and development (Jones 1980). Given the physical nature and function of giant cells, it is not unexpected that transporters, including those that transport amino acids, should be upregulated. It is also expected that xylem and

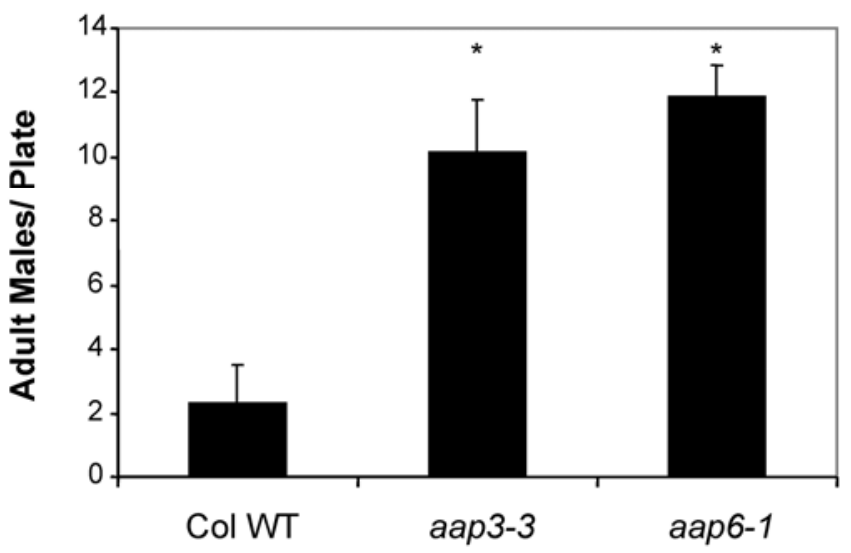

Fig. 7. Measurement of adult male nematode production in Columbia wildtype (Col WT), amino acid permease (aap)3-3, and aap6-1 plants. Seedlings of aap3-3, aap6-1, and Col WT, five seedlings per plate, were inoculated with 1,000 root-knot nematode (RKN) eggs. At 6 weeks postinoculation, the media and root material were collected from the plate and boiled in acid fuchsin stain. Nematodes were collected on filter paper and the number of adult males was counted. Significantly higher numbers of males were produced on the aap3-3 and aap6-1 mutants as compared with the Col WT. $P$ values were calculated using the Student's $t$ test. $(*=P<0.05)$.

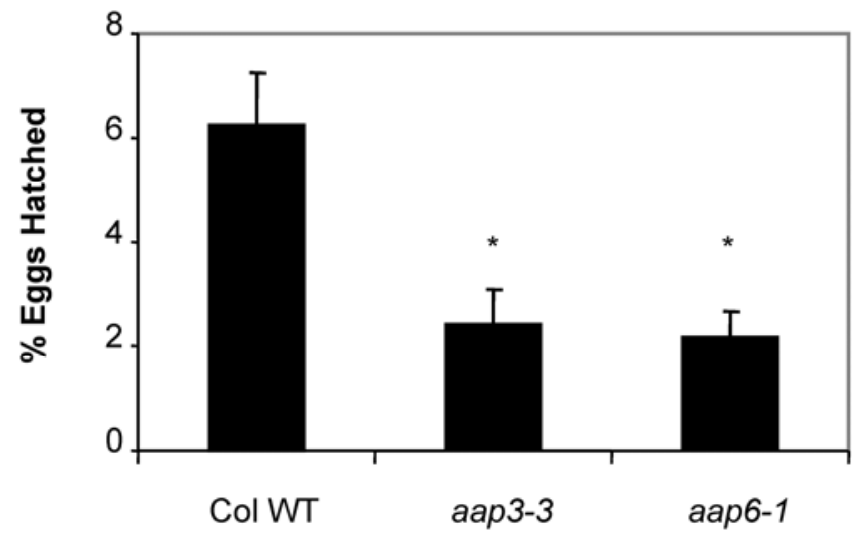

Fig. 8. Comparison of egg hatching in egg masses produced on Columbia wild-type (Col WT), amino acid permease (aap)3-3, and aap6-1 plants. Individual egg masses were isolated from the roots of Col WT, aap3-3, and aap6-1 and placed in wells of Pluronic gel. After 3 days, the number of second-stage juveniles (J2) hatched from their egg and emerged from the egg mass was counted as well as the number of unhatched eggs inside the egg mass. Significantly reduced egg hatching was observed in the aap 3-3 and aap6-1 mutants as compared with the Col WT. $P$ values were calculated using the Student's $t$ test. $(*=P<0.05)$. The number of egg masses tested $(n)$ : Col WT $(n=28)$, aap3-3 $(n=27)$, and aap6-1 $(n=26)$. 
phloem tissues and their associated companion cells surrounding and supporting the giant cells should also exhibit upregulation of transporters. $A A P 3$ and $A A P 6$ are just two of several amino acid transporters whose expression is upregulated during nematode infestation. Other upregulated AAAP family members include AtAAP7, AtAUX1, and AtLAX2 (Hammes et al. 2005). AtAUX1 transports auxin, which is also the putative function of AtLAX2, but not amino acids (Yang et al. 2006) and it is thought that auxin plays an important role in feeding site development (Barcala et al. 2010; Goverse et al. 2000). AtAAP7 remains uncharacterized, although attempts have been made to detect its amino acid transport capability and its expression (Okumoto et al. 2002). Amino acid transporters from other families are also induced by RKN infestation, including AtCAT6, a member of the APC family of amino acid transporters; however, the knockout of AtCAT6 does not display a nematode infection phenotype (Hammes et al. 2006). Interestingly, expression of AtANT1 is reduced under nematode infestation; therefore, antl mutants may have increased susceptibility to nematodes in correlation with the results of the aphid study (Hammes et al. 2005; Hunt et al. 2006). Further analysis of nematode parasitism on other amino acid transporter mutants and combinations of multiple mutations should aid in elucidating how the nematode manipulates the complex host amino acid transport system to sustain itself.

The role of AAP3 and AAP6 in plant amino acid transport.

Both AAP 3 and AAP6 are expressed in the vasculature of the plant in both root and aerial tissues (Hammes et al. 2005; Okumoto et al. 2002, 2004). They are also both believed to be loading amino acids into the phloem but from different sources, AAP3 from the apoplast and AAP6 from the xylem (Hunt et al. 2010; Okumoto et al. 2002, 2004). Analysis of the amino acid content of sieve element sap of aap6 mutant plants showed lower total amino acid concentration and lower amounts of lysine, leucine, phenylalanine, and aspartic acid, giving an in planta profile of the amino acids transported by AAP6 (Hunt et al. 2010). Interestingly, lysine, leucine, and phenylalanine are essential amino acids and, therefore, would be required by the nematode.
Here, we describe a root architecture phenotype for the aap3-3 mutant, which has a longer primary root with more lateral roots (Fig. 2). We observe this phenotype under short-day conditions, which may explain why it was not described in the previous characterization of aap3 mutants (Okumoto et al. 2004). However, we do not observe any changes in root architecture with the aap6-1 mutant. In addition, both $A A P 3$ and $A A P 6$ are expressed in flowers (Okumoto et al. 2002, 2004). However, we do not observe any phenotypes related to flowering or seed set in the aap3-3 and aap6-1 mutants in our growth conditions (data not shown), unlike a previous report of larger seed in an aap6 mutant (Hunt et al. 2010). There likely is overlap in the function and expression of amino acid transporters. Especially considering the broad spectrum of amino acids transported by each transporter and the fact that there are around 60 amino acid transporters in Arabidopsis, it is conceivable that mutants in individual transporter genes would lack phenotypes. Just in the AAP subfamily of Arabidopsis, AtAAP1, AtAAP2, and AtAAP5 are also expressed in roots and might compensate for the loss of AAP6 (Fischer et al. 1995). Both AtAAPl and AtAAP5 are involved in the uptake of amino acids into the root (Lee et al. 2007; Svennerstam et al. 2008, 2011). AtAAP2 is a particularly interesting candidate because it is localized to the vasculature (Hirner et al. 1998) like AAP3 and AAP6. However, we do not observe any effect on nematode infestation using aap 2 mutant plants (data not shown).

\section{The role of $A A P 3$ and $A A P 6$ in nematode infection.}

Nematode infestation levels, as assessed by the number of adult females producing egg masses, are significantly lower on the aap3 and aap6 mutants (Fig. 3). However, it appears that the underlying mechanisms for this reduction in infestation differ between AAP3 and AAP6. First, the carrying capacities of aap3-3 and aap6-1 differ widely. The aap3-3 mutant fails to support the same number of nematodes as the wild-type control even at the lowest level of inoculation, suggestive of an inhibitory effect in the roots (Fig. 4). Considering the increased root mass of the aap3-3 mutant (Fig. 2), which has more potential infection sites, the reduced carrying capacity is intriguing. In contrast, the carrying capacity of the aap6-1 mutant
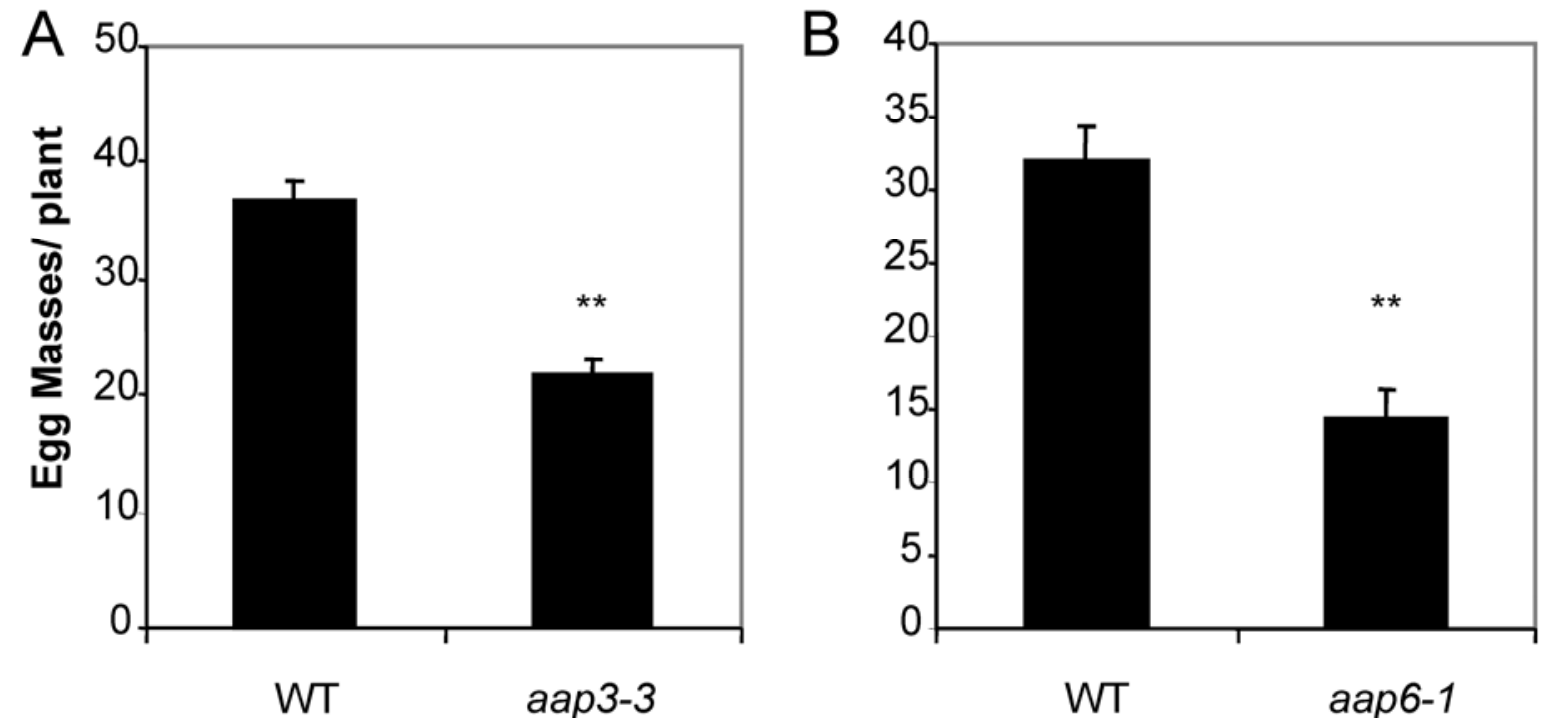

Fig. 9. Infectivity assessment of juveniles propagated on Columbia wild-type (Col WT), amino acid permease (aap)3-3, and aap6-1 plants. Synchronized hatches of juveniles from eggs isolated from Col WT and A, aap3-3 and B, aap6-1 mutants were used in inoculate tobacco ('Samsun'). The number of egg masses produced was assessed 7 weeks postinoculation by Phloxine B staining of the tobacco roots. Infectivity was significantly reduced for nematodes propagated on the aap3-3 and aap6-1 mutants as compared with those from the Col WT. $P$ values were calculated using the Student's $t$ test. (** $=P<0.001$ ). The number of plants tested $(n)$ : A, Col WT $(n=18)$ and aap3-3 $(n=15) ; \mathbf{B}, n=8$. 
does not differ from the wild type until the higher levels of inoculation, at which point its capacity saturates, indicative of host resource depletion (Fig. 4). Second, the early stages of infection of the aap3-3 mutant are altered, unlike in the aap6-1 mutant. On day 4 after infection with RKN J2, aap3-3, aap6-1, and the wild-type control had equal numbers of $\mathrm{J} 2$ inside of the roots; however, by day 6 , there were significantly fewer $\mathbf{J} 2$ inside of the aap3-3 roots (Fig. 6A). Significantly increased numbers of $\mathrm{J} 2$ are likely leaving the roots of aap3-3 plants (Fig. 6B). These results are suggestive of a repellant or inhibitory effect, which acts early in the infection process but after initial penetration of the aap3-3 root. Third, while the expression of both genes is upregulated under nematode infection, there are differences in their expression patterns. AAP6 is significantly induced in whole infected roots and even more so in individual knots as compared with the rest of the root, whereas it appears that the expression of $A A P 3$ is up across the infected root but expression is very low in the knots and giant cells (Fig. 5) (Barcala et al. 2010; Hammes et al. 2005). Furthermore,
pAAP6:GUS studies show that AAP6 is expressed throughout the gall, including the giant cells (Hammes et al. 2005). Therefore, it seems that, during nematode infestation, expression of $A A P 6$ is upregulated and may enhance the transport of amino acids into the feeding site. In contrast, $A A P 3$ expression is induced throughout the root, where it may serve to increase the amino acid level coming into vascular tissue supplying the knots. Because we do not observe an additive or synergistic effect on nematode infestation in the aap3-3/aap6-1 double mutant, we believe it is possible that these transporters are acting in a coordinated fashion, where AAP3 loads amino acids into the vasculature and AAP6 then moves these amino acids into the feeding site.

There are also similarities between nematodes on aap 3 and aap6 mutant plants in regard to the later stages of nematode infestation and the resulting progeny. Both the aap3-3 and aap6-1 mutants produce significantly more adult male nematodes under standard growth conditions (Fig. 7). Female development is the default pathway for RKN, although adult male
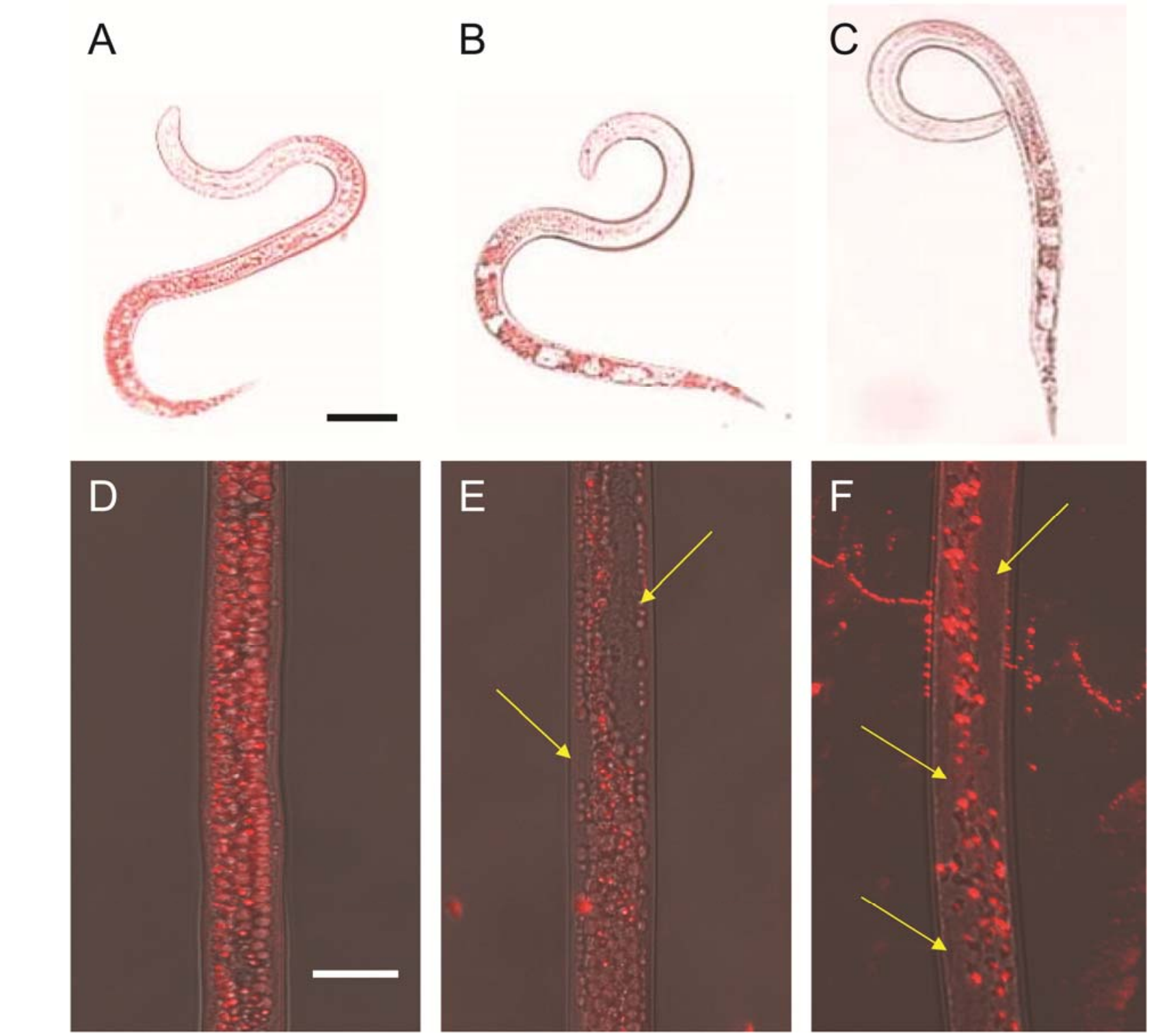

Fig. 10. Nile Red staining of lipid reserves in juveniles collected from Columbia wild-type (Col WT), amino acid permease (aap)3-3, and aap6-1 plants. Synchronized hatches of juveniles from eggs isolated from Col WT, aap3-3, and aap6-1 were stained with Nile Red at 4 days after hatching to observe the lipid reserves in the nematodes. Using confocal laser-scanning microscopy, we observed the lipid reserves in the intestine of the juveniles from A and $\mathbf{D}$, Col WT; $\mathbf{B}$ and E, aap6-1; and $\mathbf{C}$ and $\mathbf{F}$, aap3-3. Images of whole nematodes are shown in A to C and higher magnification of the intestinal tract in D to F. Arrows point to gaps in the Nile Red staining in the juveniles from aap6-1 (E) and aap3-3 (F) plants. Scale bars: A to C, $20 \mu \mathrm{m} ; \mathrm{D}$ to F, $10 \mu \mathrm{m}$. 
nematodes will develop when the host is under stress, such as high parasitic load, poor nutritional status, or physical damage (Davide and Triantaphyllou 1967, 1968; Snyder et al. 2006). Adult male development is a mechanism to optimize conditions for the survival of the remaining female nematodes. Adult males mature early and leave the root, abandoning their feeding sites and, thus, reducing the overall parasitic load and providing a better chance for the remaining female nematodes to reach maturity and reproduce. Because the carrying capacities of aap3-3 and aap6-1 are reduced as compared with the wild type (Fig. 4), the nematodes appear to be utilizing this mechanism to achieve a sustainable level of infestation.

The disruption of $A A P 3$ and AAP6 also negatively affects the progeny produced on the mutants. Eggs laid on the aap3-3 and aap6-1 mutants have reduced juvenile emergence, which is indicative of reduced vigor because the $\mathrm{J} 2$ must break out of the eggshell and exit through the gelatinous egg mass matrix (Fig. 8). Additionally, even the $\mathrm{J} 2$ that do successfully hatch from eggs produced on the aap3-3 and aap6-1 mutants are less capable of infecting tobacco roots (Fig. 9). The challenge of migrating through the sand and penetrating the root seems to have a greater effect on the $\mathrm{J} 2$ from aap3-3 and aap6-1 than $\mathrm{J} 2$ produced on the wild type. Because $\mathrm{J} 2$ are nonfeeding, they must obtain their energy through the metabolism of intestinal lipid stores (McCarter et al. 2003; Reversat 1981). Nile Red has been used to monitor lipid levels in Caenorhabditis elegans, including starved nematodes, which have less Nile Ned staining in their intestines (Ashrafi et al. 2003). Through Nile Red staining of the lipids in the $\mathrm{J} 2$, we detected less lipid reserves stored in the nematodes from aap3-3 and aap6-1 (Fig. 10). Because the progeny are dependent on the nutrition of the female, it is plausible that there is reduced amino acid flow to the progeny on the aap3-3 and aap6-1 mutants, leading to the lower lipid energy reserves, which result in the lack of vigor. A metabolic connection between amino acids and lipids has been demonstrated in C. elegans, where the monomethyl branchedchain fatty acids are synthesized using the catabolites of the branched-chain amino acids (i.e. leucine, valine, and isoleucine) (Kniazeva et al. 2004). These monomethyl branchedchain fatty acids are essential for $C$. elegans development, because RNAi suppression of a biosynthesis enzyme leads to developmental arrest in the first larval stage (Kniazeva et al. 2004). Leucine is one of the amino acids significantly reduced in the phloem sap of aap6 mutants (Hunt et al. 2010); therefore, perhaps it is the reduced availability of leucine that is affecting the lipid reserves in the nematode progeny.

\section{MATERIALS AND METHODS}

\section{Plant material.}

A. thaliana was grown on medium consisting of $2 \%$ sucrose, $0.3 \%$ Gamborg's basal salts, and $0.6 \%$ Phytagel, pH 6.1, and kept in a short-day chamber $\left(23^{\circ} \mathrm{C}\right.$ for $8 \mathrm{~h}$ of light and $16 \mathrm{~h}$ of darkness, $70 \mu \mathrm{mol} \mathrm{s} \mathrm{m}^{-1}$ ) for all experiments. The aap3-3 (SALK_148822) and aap6-1 (SALK_013231) mutants were identified by PCR as described (Alonso et al. 2003). The aap3-2 mutant (Okumoto et al. 2004) was a gift from W. Frommer. The aap3-3/aap6-1 double mutant was generated by crossing the two mutants and subsequently PCR genotyping the F2 generation to identify the double homozygous lines.

Nicotiana tabacum 'Samsun' was grown in a greenhouse. Seed were germinated in soil for 2 weeks prior to transfer to sand.

\section{RT-PCR.}

RNA was isolated from 8-day-old seedlings grown as described above by using the RNeasy Plant Mini kit (Qiagen, Va- lencia, CA, U.S.A.) following the manufacturer's protocol. Total RNA (500 ng) was used as the starting template for reverse transcription using the SuperScript III First-Strand Synthesis System (Invitrogen, Carlsbad, CA, U.S.A.) with oligo dT. Thirty cycles of PCR were performed with diluted cDNA $(1: 10)$ and the following primers: AAP6-fwd 5'-GTGGCGGTG AGCACGTAAGA-3', AAP6-rev 5'-GCAGACATTGGCAAAG TCGA-3', AAP3-fwd 5'-ACCAAGTCTATTGCCAACCTC-3', AAP3-rev 5'-TCAGTATTCGCTTCGAAATGGC-3', UBP22fwd 5'-GCCAAAGCTGTGGAGAAAAG-3', and UBP22-rev 5'-TGTTTAGGCGGAACGGATAC-3'.

\section{Nematode culture and infestation assay.}

The dixenic $M$. incognita population was maintained on Arabidopsis ecotype Columbia as previously described (Hammes et al. 2005). For the nematode infestation assays, Arabidopsis seed were sterilized and sown at five seeds per plate on the media described above. The plates were wrapped in parafilm and placed at a $45^{\circ}$ angle in a short-day chamber $\left(23^{\circ} \mathrm{C}\right.$ for $8 \mathrm{~h}$ of light and $16 \mathrm{~h}$ of darkness, $\left.70 \mu \mathrm{mol} \mathrm{s} \mathrm{s}^{-1} \mathrm{~m}^{-2}\right)$. After 3 weeks, 1,000 sterilely propagated $M$. incognita eggs were inoculated on the roots of Arabidopsis for each plate. The plates were then wrapped with micropore tape, placed at an angle inside clear acrylic boxes, lined on the bottom with damp felt to prevent the drying of the media, and returned to the short-day chamber. After 8 weeks of infection, the number of females producing egg masses was counted for each plate. Each trial consisted of 10 plates per genotype.

$\mathrm{J} 2$ were obtained by placing freshly collected RKN eggs on sterile $25-\mu \mathrm{m}$ wire mesh suspended just above a layer of water inside a sterile petri dish kept at $26^{\circ} \mathrm{C}$. $\mathrm{J} 2$ that hatched from their eggs fell into the water below the mesh over the course of several days. The water containing the $\mathrm{J} 2$ was collected and the wire mesh was transferred to a new petri dish to obtain synchronized hatches of $\mathrm{J} 2$.

\section{Carrying-capacity assay.}

Three-week old seedlings of the Columbia wild type, aap3-3, and aap6-1 were grown as described above. Freshly hatched, synchronized $\mathrm{J} 2$ were added in increasing amounts (300, 600, or 900) to each plate. Each data point consisted of between 7 to 10 plates. The number of resulting egg masses was counted at 8 weeks postinoculation.

\section{GUS staining.}

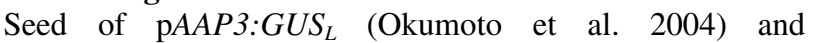
pAAP6:GUS (Hammes et al. 2005) transgenic Arabidopsis were grown on plates as described above. The plates were inoculated with 1,000 RKN eggs as described earlier. At 1 and 4 weeks postinoculation, the root tissue was harvested and placed in 6-well microtiter plates with $4 \mathrm{ml}$ of GUS staining solution $\left(0.1 \mathrm{M} \mathrm{NaPO}_{4}\right.$ at $\mathrm{pH} 7.0,0.5 \mathrm{mM} \mathrm{K} 3\left[\mathrm{Fe}(\mathrm{CN})_{6}\right], 0.5 \mathrm{mM} \mathrm{K} 4$ [Fe $\left.(\mathrm{CN})_{6}\right], 10 \mathrm{mM}$ EDTA, $0.1 \%$ Triton X-100, and 5-bromo4-chloro-3-indoyl- $\beta$-glucuronide at $1 \mathrm{mg} / \mathrm{ml}$ dissolved in dimethyl sulfoxide) for $16 \mathrm{~h}$ at $37^{\circ} \mathrm{C}$ (Jefferson et al. 1987). Stained roots were examined using a Nikon SMZ 1500 dissecting microscope.

Sections of knots produced by RKN infection were produced by first GUS staining hand-dissected knots for $16 \mathrm{~h}$ as described above and then dehydrating through a water/ethanol series: 10, 25, 50, 75, and 95\% for $1 \mathrm{~h}$ each. Fixed dehydrated knots were then infiltrated in a series of Histoclear in ethanol: 50, 75, and 95\% Histoclear for $2 \mathrm{~h}$ each and $100 \%$ Histoclear overnight. Finally, knots were infiltrated in 1:1 Histoclear/ Paraplast tissue-embedding medium for $8 \mathrm{~h}$ at $56^{\circ} \mathrm{C}$ and then infiltrated in Paraplast tissue-embedding medium for $96 \mathrm{~h}$. Knot sections ( $20 \mu \mathrm{m}$ thick) were generated by a wax microtome 
and examined using a Nikon Eclipse E800 microscope with bright-field optics.

\section{Early infection assays.}

Twenty 6-day-old seedlings, each with only a primary root, were arranged in a circle on a fresh plate with their roots pointing inward and allowed to recover for $24 \mathrm{~h}$. Each plate was then inoculated with $1,000 \mathrm{~J} 2$ in the center of the circle of roots. Every $48 \mathrm{~h}$ for 6 days, the seedlings from one plate for each genotype were stained with acid fuchsin to visualize the $\mathrm{J} 2$ which had infected the roots. The acid fuchsin staining was carried out as follows. The seedlings were placed in $1.5 \%$ sodium hypochlorite for $4 \mathrm{~min}$. The seedlings were then washed in water for $15 \mathrm{~min}$ and transferred to a 1:30 dilution of acid fuchsin staining solution ( $3.5 \mathrm{~g}$ in 1 liter of $25 \%$ acetic acid) (Sigma-Aldrich, St. Louis). The acid fuchsin staining solution with the seedlings was brought to a boil in a microwave and then cooled to room temperature. The seedlings were briefly destained in water and then placed in acidified glycerol. The number of stained $\mathrm{J} 2$ inside each root was counted.

To capture any $\mathrm{J} 2$ leaving roots, the assay was set up as described above, except that the seedlings were removed after 4 days of infection. Each seedling was transferred to a single well containing ice-cold, liquid-state 23\% Pluronic F127 gel (BASF, Florham Park, NJ, U.S.A.). The Pluronic gel was solidified at room temperature and then multi-well plates were placed at $26^{\circ} \mathrm{C}$ for $48 \mathrm{~h}$. The Pluronic gel was liquefied at $4{ }^{\circ} \mathrm{C}$ for $15 \mathrm{~h}$ and then the seedlings were removed and stained with acid fuchsin as described above to detect the number of $\mathrm{J} 2$ inside the root. The $\mathrm{J} 2$ that had left the roots were counted for each well of Pluronic gel. The percentage of $\mathrm{J} 2$ retained was calculated for each seedling from the number of $\mathrm{J} 2$ stained by acid fuchsin inside the root and the number which were left in the well of Pluronic gel.

\section{Adult male production.}

Nematode infestation was performed as described above. Eight weeks postinoculation, the roots and media from each plate were placed in flask with $150 \mathrm{ml}$ of water, $5 \mathrm{ml}$ of acid fuchsin stain, and $1 \mathrm{ml}$ of $0.5 \mathrm{M}$ EDTA. The flask was then heated in the microwave until the solution was boiling and the media had melted. The solution was then poured through a sieve, to capture the root material, and the nematodes were collected by vacuum filtration on glass filter paper. The number of adult males was counted for each plate.

\section{J2 emergence assay.}

Nematode infestation was performed as described above. At 7 weeks postinoculation, individual females with egg masses were isolated and placed in individual wells of liquefied $23 \%$ Pluronic F127 gel (BASF). The Pluronic gel was solidified at room temperature and the multi-well plate was incubated at $26^{\circ} \mathrm{C}$ for 3 days. The multi-well plate was then incubated at $4^{\circ} \mathrm{C}$ for $15 \mathrm{~h}$ to liquefy the Pluronic gel. The female and egg mass were removed and vortexed in a $1.5-\mathrm{ml}$ tube with $5 \%$ bleach to disrupt the egg mass and release the eggs. The eggs that remained unhatched for each egg mass were then counted. The number of juveniles that had hatched and emerged from the egg mass and were left behind in each well of Pluronic gel were also counted. The percentage of eggs hatched was then calculated for each egg mass.

\section{Tobacco infection.}

Tobacco seed were germinated in soil and, after 2 weeks, transferred to individual pots of sand for an additional week. Eggs were collected from infected Columbia wild type, aap3-3, and aap6-1 at 8 weeks postinoculation. The eggs were set for hatching as described above and the $\mathrm{J} 2$ that hatched on days 7 and 8 were used for the tobacco infection. In all, $200 \mathrm{~J} 2$ were applied to each tobacco plant. At 7 weeks postinoculation, the tobacco roots were gently rinsed in water to remove the sand and then stained with $0.05 \%$ Phloxine B (Sigma-Aldrich) for $15 \mathrm{~min}$. Roots were then rinsed four times with water. The egg masses stained a dark red color and were counted.

\section{Nile Red staining.}

Eggs were isolated from RKN-infected Columbia wild-type, aap3-3, and aap6-1 plants and set for hatching as previously described. The wire mesh with the eggs was removed on day 7 , leaving behind the $\mathrm{J} 2$ which had already hatched out, and transferred to a fresh petri dish to collect a synchronous batch of $\mathrm{J} 2$ hatched out on days 7 and 8 . The synchronously hatched juveniles were collected and stained with Nile Red (Polysciences, Warrington, PA, U.S.A.) at $1 \mu \mathrm{g} / \mathrm{ml}$ for $30 \mathrm{~min}$. Confocal microscopy was performed using a Zeiss LSM 510 META NLO, exciting with a HeNe 543-nm laser and using a longpass filter $(560 \mathrm{~nm})$. Sixty nematodes from each line were scored for Nile Red staining pattern.

\section{ACKNOWLEDGMENTS}

H. Marella conducted all experiments described in the article and was responsible for the writing of this manuscript as well. E. Nielsen, D. P. Schachtman, and C. G. Taylor were responsible for oversight of the project, experimental design, and review of the manuscript. Taylor is the corresponding author on this article and responsible for final approval of this manuscript. This research was funded, in part, by the National Science Foundation (numbers 0344265, 0821954, and 1104334). We thank V. Carballo, B. Andrews, and N. Breakfield for their technical assistance; T. Fester and R. H. Berg for their help with the microscopy; and W. Frommer and S. Okumoto for providing the aap3-2 and pAAP3:GUS $S_{L}$ seed.

\section{LITERATURE CITED}

Alonso, J. M., Stepanova, A. N., Leisse, T. J., Kim, C. J., Chen, H., Shinn, P., Stevenson, D. K., Zimmerman, J., Barajas, P., Cheuk, R., Gadrinab, C., Heller, C., Jeske, A., Koesema, E., Meyers, C. C., Parker, H., Prednis, L., Ansari, Y., Choy, N., Deen, H., Geralt, M., Hazari, N., Hom, E., Karnes, M., Mulholland, C., Ndubaku, R., Schmidt, I., Guzman, P., Aguilar-Henonin, L., Schmid, M., Weigel, D., Carter, D. E., Marchand, T., Risseeuw, E., Brogden, D., Zeko, A., Crosby, W. L., Berry, C. C., and Ecker, J. R. 2003. Genome-wide insertional mutagenesis of Arabidopsis thaliana. Science 301:653-657.

Ashrafi, K., Chang, F. Y., Watts, J. L., Fraser, A. G., Kamath, R. S., Ahringer, J., and Ruvkun, G. 2003. Genome-wide RNAi analysis of Caenorhabditis elegans fat regulatory genes. Nature 421:268-272.

Barcala, M., García, A., Cabrera, J., Casson, S., Lindsey, K., Favery, B. García-Casado, G., Solano, R., Fenoll, C., and Escobar, C. 2010. Early transcriptomic events in microdissected Arabidopsis nematode-induced giant cells. Plant J._61:698-712

Bird, D. M. 2004. Signaling between nematodes and plants. Curr. Opin. Plant Biol. 7:372-376.

Caillaud, M.-C., Dubreuil, G., Quentin, M., Perfus-Barbeoch, L., Lecomte, P., de Almeida Engler, J., Abad, P., Rosso, M.-N., and Favery, B. 2008 Root-knot nematodes manipulate plant cell functions during a compatible interaction. J. Plant Physiol. 165:104-113.

Chang, A. B., Lin, R., Studley, W. K., Tran, C. V., and Saier, M. H. 2004 Phylogeny as a guide to structure and function of membrane transport proteins. Mol. Membr. Biol. 21:171-181.

Davide, R. G., and Triantaphyllou, A. C. 1967. Influence of the environment on development and sex differentiation of root-knot nematodes II Effect of host nutrition. Nematologica 13:111-117.

Davide, R. G., and Triantaphyllou, A. C. 1968. Influence of the environment on development and sex differentiation of root-knot nematodes III. Effect of foliar application of maleic hydrazide. Nematologica 14:37-46.

Fischer, W. N., Kwart, M., Hummel, S., and Frommer, W. B. 1995. Substrate specificity and expression profile of amino acid transporters (AAPs) in Arabidopsis. J. Biol. Chem. 270:16315-16320.

Fischer, W. N., Loo, D. D. F., Koch, W., Ludewig, U., Boorer, K. J., Tegeder, M., Rentsch, D., Wright, E. M., and Frommer, W. B. 2002. 
Low and high affinity amino acid $\mathrm{H}^{+}$-cotransporters for cellular import of neutral and charged amino acids. Plant J. 29:717-731.

Goverse, A., Overmars, H., Engelbertink, J., Schots, A., Bakker, J., and Helder, J. 2000. Both induction and morphogenesis of cyst nematode feeding cells are mediated by auxin. Mol. Plant-Microbe Interact. 13:1121-1129.

Hammes, U. Z., Schachtman, D. P., Berg, R. H., Nielsen, E., Koch, W., McIntyre, L. M., and Taylor, C. G. 2005. Nematode-induced changes of transporter gene expression in Arabidopsis roots. Mol. Plant-Microbe Interact. 18:1247-1257.

Hammes, U. Z., Nielsen, E., Honaas, L. A., Taylor, C. G., and Schachtman, D. P. 2006. AtCAT6, a sink-tissue-localized transporter for essential amino acids in Arabidopsis. Plant J. 48:414-426.

Hirner, B., Fischer, W. N., Rentsch, D., Kwart, M., and Frommer, W. B. 1998. Developmental control of H+/amino acid permease gene expression during seed development of Arabidopsis. Plant J. 14:535-544.

Hunt, E. J., Pritchard, J., Bennett, M. J., Zhu, X., Barrett, D. A., Allen, T., Bale, J., and Newbury, H. J. 2006. The Arabidopsis thaliana/Myzus persicae model system demonstrates that a single gene can influence the interaction between a plant and a sap-feeding insect. Mol. Ecol. 15:42034213.

Hunt, E., Gattolin, S., Newbury, H. J., Bale, J. S., Tseng, H. M., Barrett, D. A., and Pritchard, J. 2010. A mutation in amino acid permease AAP6 reduces the amino acid content of the Arabidopsis sieve elements but leaves aphid herbivores unaffected. J. Exp. Bot. 61:55-64.

Jammes, F., Lecomte, P., de Almeida-Engler, J., Bitton, F., MartinMagniette, M. L., Renou, J. P., Abad, P., and Favery, B. 2005. Genomewide expression profiling of the host response to root-knot nematode infection in Arabidopsis. Plant J. 44:447-458.

Jefferson, R. A., Kavanagh, T. A., and Bevan, M. W. 1987. GUS fusion: $\beta$ glucuronidase as a sensitive and versatile gene fusion marker in higher plants. EMBO (Eur. Mol. Biol. Organ.) J. 6:3901-3907.

Jones, M. G. K. 1980. Host cell responses to endoparasitic nematode attack: Structure and function of giant cells and synctia. Ann. Appl. Biol. 97:353-372.

Kniazeva, M., Crawford, Q. T., Seiber, M., Wang, C., and Han, M. 2004 Monomethyl branched-chain fatty acids play an essential role in Caenorhabditis elegans development. PLoS Biol. 2:e257. Published online. doi:10.1371/journal.pbio.0020257

Lee, Y. H., Foster, J., Chen, J., Voll., L. M., Weber, A. P. M., and Tegeder, M. 2007. AAP1 transports uncharged amino acids into roots of Arabidopsis. Plant J. 50:305-319.

McCarter, J. P., Mitreva, M. D., Martin, J., Dante, M., Wylie, T., Rao, U., Pape, D., Bowers, Y., Theising, B., Murphy, C. V., Kloek, A. P., Chiapelli, B. J., Clifton, S. W., Bird, D. M., and Waterston, R. H. 2003. Analysis and functional classification of transcripts from the nematode Meloidogyne incognita. Genome Biol. 4:R26.

Okumoto, S., Schmidt, R., Tegeder, M., Fischer, W. N., Rentsch, D. Frommer, W. B., and Koch, W. 2002. High affinity amino acid transporters specifically expressed in xylem parenchyma and developing seeds of Arabidopsis. J. Biol. Chem. 277:45338-45346.
Okumoto, S., Koch, W., Tegeder, M., Fischer, W. N., Biehl, A., Leister, D Stierhof, Y. D., and Frommer, W. B. 2004. Root phloem-specific expression of the plasma membrane amino acid proton co-transporter AAP3 J. Exp. Bot. 55:2155-2168.

Puthoff, D. P., Nettleton, D., Rodermel, S. R., and Baum, T. J. 2003. Arabidopsis gene expression changes during cyst nematode parasitism revealed by statistical analyses of microarray expression profiles. Plant J. 33:911-921.

Puthoff, D. P., Ehrenfried, M. L., Vinyard, B. T., and Tucker, M. L. 2007. GeneChip profiling of transcriptional responses to soybean cyst nematode, Heterodera glycines, colonization of soybean roots. J. Exp. Bot. 58:3407-3418.

Rentsch, D., Schmidt, S., and Tegeder, M. 2007. Transporters for uptake and allocation of organic nitrogen compounds in plants. FEBS (Fed. Eur. Biochem. Soc.) Lett. 581:2281-2289.

Reversat, G. 1981. Consumption of food reserves by starved second-stage juveniles of Meloidogyne javanica under conditions including osmobiosis. Nematologica 27:207-214.

Snyder, D. W., Opperman, C. H., and Bird, D. M. 2006. A method for generating Meloidogyne incognita males. J. Nematol. 38:192-194.

Struck, C., Mueller, E., Martin, H., and Lohaus, G. 2004. The Uromyces fabae UfAAT3 gene encodes a general amino acid permease that prefers uptake of in planta scarce amino acids. Mol. Plant Pathol. 5:183189.

Svennerstam, H., Ganeteg, U., and Nasholm, T. 2008. Root uptake of cationic amino acids by Arabidopsis depends on functional expression of amino acid permease. New Phytol. 180:620-630.

Svennerstam, H., Jamtgard, S., Ahmad, I., Huss-Danell, K., Nasholm, T. and Ganeteg, U. 2011. Transporters in Arabidopsis roots mediating uptake of amino acids at naturally occurring concentrations. New Phytol. 191:459-467.

Szakasits, D., Heinen, P., Wieczorek, K., Hofmann, J., Wagner, F., Kreil, D. P., Sykacek, P., Grundler, F. M. W., and Bohlmann, H. 2009. The transcriptome of syncytia induced by the cyst nematode Heterodera schachtii in Arabidopsis roots. Plant J. 57:771-784.

Tegeder, M. 2012. Transporters for amino acids in plant cells: Some functions and many unknowns. Curr. Opin. Plant Biol. 15:315-321.

Tegeder, M., and Rentsch, D. 2010. Uptake and partitioning of amino acids and peptides. Mol. Plant 3:997-1011.

Wanasen, N., MacLeod, C. L., Ellies, L. G., and Soong, L. 2007. L-Arginine and cationic amino acid transporter $2 \mathrm{~B}$ regulate growth and survival of Leishmania amazonensis amastigotes in macrophages. Infect. Immun. 75:2802-2810.

Williamson, V. M., and Gleason, C. A. 2003. Plant-nematode interactions Curr. Opin. Plant Biol. 6:327-333.

Wipf, D., Ludewig, U., Tegeder, M., Rentsch, D., Koch, W., and Frommer, W. B. 2002. Conservation of amino acid transporters in fungi, plants and animals. Trends Biochem. Sci. 27:139-147.

Yang, Y., Hammes, U. Z., Taylor, C. G., Schachtman, D. P., and Nielsen, E. 2006. High-affinity auxin transport by the AUX1 influx carrier protein. Curr. Biol. 16:1123-1127. 